University of Nebraska - Lincoln

DigitalCommons@University of Nebraska - Lincoln

Faculty Publications from the Department of Electrical \& Computer Engineering, Department Electrical and Computer Engineering

2011

\title{
Effective Capacity Region and Optimal Power Control for Fading Broadcast Channels
}

Deli Qiao

University of Nebraska-Lincoln, dlqiao@ce.ecnu.edu.cn

M. Cenk Gursoy

University of Nebraska-Lincoln, gursoy@engr.unl.edu

Senem Velipasalar

University of Nebraska-Lincoln, velipasa@engr.unl.edu

Follow this and additional works at: https://digitalcommons.unl.edu/electricalengineeringfacpub

Part of the Electrical and Computer Engineering Commons

Qiao, Deli; Cenk Gursoy, M.; and Velipasalar, Senem, "Effective Capacity Region and Optimal Power Control for Fading Broadcast Channels" (2011). Faculty Publications from the Department of Electrical and Computer Engineering. 209.

https://digitalcommons.unl.edu/electricalengineeringfacpub/209

This Article is brought to you for free and open access by the Electrical \& Computer Engineering, Department of at DigitalCommons@University of Nebraska - Lincoln. It has been accepted for inclusion in Faculty Publications from the Department of Electrical and Computer Engineering by an authorized administrator of DigitalCommons@University of Nebraska - Lincoln. 


\title{
Effective Capacity Region and Optimal Power Control for Fading Broadcast Channels
}

\author{
Deli Qiao, Mustafa Cenk Gursoy, and Senem Velipasalar \\ Department of Electrical Engineering \\ University of Nebraska-Lincoln, Lincoln, NE 68588 \\ Email: dqiao726@huskers.unl.edu,gursoy@engr.unl.edu,velipasa@engr.unl.edu
}

\begin{abstract}
Transmission over fading broadcast channels in the presence of quality of service $(\mathrm{QoS})$ constraints is studied. Effective capacity, which provides the maximum constant arrival rate that a given service process can support while satisfying statistical QoS constraints, is employed as the performance metric. The effective capacity region achieved with superposition coding and successive interference cancellation is identified and is shown to be convex. Subsequently, optimal power control policies that achieve the boundary points of the effective capacity region are investigated, and an algorithm for the numerical computation of the optimal power adaptation schemes for the two-user case is provided. Additionally, performance attained with time-division multiplexing (TDM) of messages is studied for comparison with the optimal schemes.
\end{abstract}

\section{INTRODUCTION}

Fading broadcast channels (BC) have been extensively studied over the years from an information-theoretic point of view. For instance, $\mathrm{Li}$ and Goldsmith in [1] characterized the ergodic capacity region and determined the optimal resource allocation policies. They showed that the capacity region, which is achieved by superposition coding and successive decoding whose order is determined by the effective noise levels, is convex. They provided an algorithm to determine the optimal power allocation in each fading state. The same authors in [2] studied the outage capacity. In [3], Seong et al. obtained a throughput optimal scheduling policy that incorporates the queue state vector and achieves the points on the boundary surface of the ergodic capacity region.

In this paper, we study the throughput in fading broadcast channels in the presence of statistical quality of service (QoS) constraints. For this analysis, we employ the concept of effective capacity [4], which can be seen as the maximum constant arrival rate that a given time-varying service process can support while satisfying statistical QoS guarantees. Effective capacity formulation uses the large deviations theory and incorporates the statistical QoS constraints by capturing the rate of decay of the buffer occupancy probability for large queue lengths. Effective capacity has recently been considered as a performance metric in multiuser scenarios for systems operating under queuing constraints. For instance, Liu et al. in [7] studied a model in which two users collaborate to send their data to a common destination node using frequencydivision multiplexing. In this setting, effective capacity is used

\footnotetext{
${ }^{1}$ This work was supported by the National Science Foundation under Grants CNS-0834753, and CCF-0917265.
}

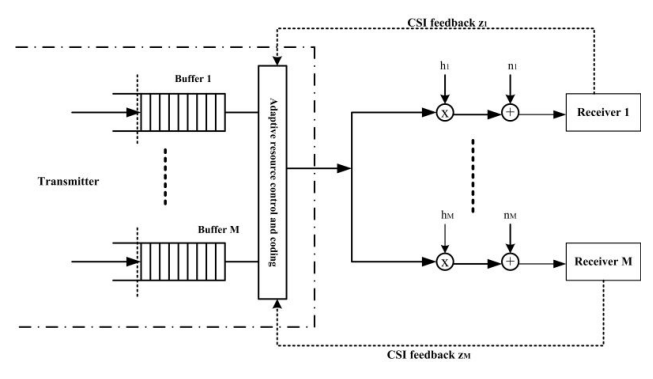

Fig. 1. The general system model.

to identify the achievable rate regions under QoS constraints. While this work investigated a cooperative multiple-access scenario, references [8] and [9] considered fading broadcast channels. Du and Zhang in [8] obtained the optimal resource allocation policies in time-division-based wireless downlink tranmissions. Balasubramanian and Miller in [9] derived the optimal time division strategy for each channel state but considered fixed power transmissions.

As outlined above, most studies in multiuser settings have studied the performance achieved with orthogonal signaling through time-division or frequency-division multiplexing. In this paper, we consider the more general and informationtheoretically optimal schemes of superposition coding and successive interference cancellation (or equivalently successive decoding). We assume that both the transmitter and the receivers have perfect channel side information (CSI). We determine the effective capacity region and explore the optimal power control policies that achieve the points on the boundary. We also discuss a time-division multiplexing strategy for comparison.

\section{SySTEM MODEL}

As shown in Figure 1, we consider a downlink scenario in which the transmitter wishes to send messages to $M$ users with individual QoS constraints under a total average power limitation. It is assumed that the data sequences generated for each receiver are divided into frames of duration $T$ and are initially stored in individual buffers before they are transmitted over the wireless channel. The discrete-time signal at the $j^{\text {th }}$ receiver in the $i^{\text {th }}$ symbol duration is given by

$$
Y_{j}[i]=h_{j}[i] X[i]+n_{j}[i], \quad j=1, \ldots, M \text { and } i=1,2, \ldots
$$


where $M$ is the number of users, $X[i]$, composed of $M$ independent information sources, denotes the complex-valued channel input, and $h_{j}[i]$ represents the fading coefficient of the $j$ th user. We denote the magnitude-square of the fading coefficients by $z_{j}[i]=\left|h_{j}[i]\right|^{2}$. The channel input is subject to the average energy constraint $\mathbb{E}\left\{|X[i]|^{2}\right\} \leq \bar{P} / B$ where $B$ is the bandwidth available in the system and hence $\bar{P}$ is the average power constraint (assuming that the symbol rate is $B$ complex symbols per second). $Y_{j}[i]$ is the channel output at the $j$ th receiver. Above, $n_{j}[i], j=1,2, \ldots, M$ are zero-mean, circularly symmetric, complex Gaussian random variables with variances $\mathbb{E}\left\{\left|n_{j}[i]\right|^{2}\right\}=N_{j}$. The additive Gaussian noise samples $\left\{n_{j}[i]\right\}$ are assumed to form an independent and identically distributed (i.i.d.) sequence.

We denote the average transmitted signal to noise ratio with respect to receiver 1 as SNR $=\frac{\bar{P}}{N_{1} B}$. Also, we denote the instantaneous transmit power for user $j$ as $P_{j}[i]$. Now, the instantaneous transmitted SNR level for receiver $j$ becomes $\mu_{j}[i]=\frac{P_{j}[i]}{N_{j} B}$. Then, the average power constraint at the transmitter is equivalent to

$$
\mathbb{E}\left\{\sum_{j=1}^{M} \gamma_{j} \mu_{j}[i]\right\} \leq \mathrm{SNR}
$$

where we have defined the ratio $\gamma_{j}=\frac{N_{j}}{N_{1}}$.

\section{A. Ergodic Capacity Region}

The capacity region of this fading broadcast channel, achieved by superposition coding at the transmitter and successive interference cancellation at the receivers, is [1]:

$$
\begin{aligned}
\mathcal{R}_{\mathrm{BC}} & =\left\{\left(R_{1}, \ldots, R_{M}\right): R_{j} \leq\right. \\
& \left.B \mathbb{E}_{\mathbf{z}}\left\{\log _{2}\left(1+\frac{\mu_{j}(\mathbf{z}) z_{j}}{1+\sum_{k=1}^{M} \mu_{k}(\mathbf{z}) \frac{\gamma_{k} z_{j}}{\gamma_{j}} \mathbf{1}\left[\gamma_{j} / z_{j}>\gamma_{k} / z_{k}\right]}\right)\right\}\right\}
\end{aligned}
$$

where $\mathbf{1}[\cdot]$ is an indicator function (i.e., $\mathbf{1}[x]=1$ if $x$ is true, and zero otherwise), $\mu_{k}(\mathbf{z}) \frac{\gamma_{k} z_{j}}{\gamma_{j}}=\frac{\left.P_{k} i\right] z_{j}}{N_{j} B}$ denotes the interference level caused by the message for user $k, \mu=$ $\left(\mu_{1}(\mathbf{z}), \ldots, \mu_{M}(\mathbf{z})\right)$ can be regarded as the power allocation policies that need to satisfy the average power constraint (1), and $\mathbf{z}=\left(z_{1}, \cdots, z_{M}\right)$ is a random vector comprised of the magnitude squares of the channel coefficients. Also, note that $\gamma_{j} / z_{j}>\gamma_{k} / z_{k}$ is equivalent to $\frac{N_{j}}{z_{j}}>\frac{N_{k}}{z_{k}}$, which is used in determining the decoding order for the users. More specifically, the permutation order for successful decoding is given by $\frac{N_{\pi}(1)}{z_{\pi}(1)}<\frac{N_{\pi}(2)}{z_{\pi}(2)} \cdots<\frac{N_{\pi}(M)}{z_{\pi}(M)}$, which is the order of effective noise level.

The ergodic capacity region $\mathcal{R}_{\mathrm{BC}}$ is convex [1]. For any channel state vector $\mathbf{z}$, the decoding order is fixed and the maximum instantaneous rate for user $j$ is

$R_{j}=B \log _{2}\left(1+\frac{\mu_{j}(\mathbf{z}) z_{j}}{1+\sum_{k=1}^{M} \mu_{k}(\mathbf{z}) \frac{\gamma_{k} z_{j}}{\gamma_{j}} \mathbf{1}\left[\gamma_{j} / z_{j}>\gamma_{k} / z_{k}\right]}\right)$ bits/s

\section{B. Time-Division Multiplexing}

For simplicity, we assume that the time division strategy is fixed prior to transmission and hence does not vary with fading. Let $\delta_{j} \in[0,1]$ denote the fraction of time allocated to user $j$. Hence, in each frame, $\delta_{j}$ fraction of the time is dedicated to the transmission of the message intended for user $j$. Note that we have $\sum_{j=1}^{M} \delta_{j}=1$. Suppose we also have allocated a fraction $\rho_{j} \in[0,1]$ of the total average power to user $j$. Also, $\sum_{j=1}^{M} \rho_{j}=1$. Then, the maximum instantaneous service rate for user $j$ in the allocated time slot is given by

$$
R_{j}=B \log _{2}\left(1+\mu_{j}(\mathbf{z}) z_{j}\right) \text { bits/s }
$$

where $\mu_{j}$ satisfies the following average power constraint

$$
\mathbb{E}\left\{\gamma_{j} \mu_{j}(\mathbf{z})\right\} \leq \rho_{j} \text { SNR. }
$$

\section{PRELIMINARIES}

In [4], Wu and Negi defined the effective capacity as the maximum constant arrival rate $^{2}$ that a given service process can support in order to guarantee a statistical QoS requirement specified by the QoS exponent $\theta$. If we define $Q$ as the stationary queue length, then $\theta$ is the decay rate of the tail distribution of the queue length $Q$ :

$$
\lim _{q \rightarrow \infty} \frac{\log P(Q \geq q)}{q}=-\theta .
$$

Therefore, for large $q_{\max }$, we have the following approximation for the buffer violation probability: $P\left(Q \geq q_{\max }\right) \approx$ $e^{-\theta q_{\max }}$. Hence, while larger $\theta$ corresponds to more strict QoS constraints, smaller $\theta$ implies looser QoS guarantees. Similarly, if $D$ denotes the steady-state delay experienced in the buffer, then $P\left(D \geq d_{\max }\right) \approx e^{-\theta \xi d_{\max }}$ for large $d_{\max }$, where $\xi$ is determined by the arrival and service processes [6]. Since the average arrival rate is equal to the average departure rate when the queue is in steady-state, effective capacity can also be seen as the maximum throughput in the presence of such constraints. The effective capacity is given by

$$
C(\theta)=-\lim _{t \rightarrow \infty} \frac{1}{\theta t} \log _{e} \mathbb{E}\left\{e^{-\theta S[t]}\right\} \quad \text { bits/s, }
$$

where the expectation is with respect to $S[t]=\sum_{i=1}^{t} s[i]$, which is the time-accumulated service process. $\{s[i], i=$ $1,2, \ldots\}$ denote the discrete-time stationary and ergodic stochastic service process.

In this paper, in order to simplify the analysis while considering general fading distributions, we assume that the fading coefficients stay constant over the frame duration $T$ and vary independently for each frame. In this scenario, $s[i]=T R[i]$, where $R[i]$ is the instantaneous service rate in the $i$ th frame duration $[i T ;(i+1) T)$. Then, (7) can be written as

$$
C(\theta)=-\frac{1}{\theta T} \log _{e} \mathbb{E}_{\mathbf{z}}\left\{e^{-\theta T R[i]}\right\} \quad \text { bits/s, }
$$

which is obtained using the fact that the instantaneous rates $\{R[i]\}$, which are in general functions of $\mathbf{z}$, vary independently

${ }^{2}$ For time-varying arrival rates, effective capacity specifies the effective bandwidth of the arrival process that can be supported by the channel. 
from one frame to another. Note that the instantaneous service rates in fading broadcast channels are given by (3) or (4) depending on the transmission scheme.

Throughout the rest of the paper, we use the effective capacity normalized by bandwidth $B$, which is denoted by

$$
\mathrm{C}(\theta)=\frac{C(\theta)}{B} \quad \text { bits/s/Hz. }
$$

\section{EFFective CAPAcity Region}

In this section, we first identify the effective capacity region and investigate the optimal power adaptation strategies when superposition coding with successive decoding is employed. Subsequently, we characterize the effective throughput region achieved with time-division multiplexing (TDM).

\section{A. Superposition Coding with Successive Decoding}

Suppose that $\Theta=\left(\theta_{1}, \cdots, \theta_{M}\right)$ is a vector composed of the QoS constraints of $M$ users. Let $\mathrm{C}(\Theta)=$ $\left(C_{1}\left(\theta_{1}\right), \cdots, C_{M}\left(\theta_{M}\right)\right)$ denote the vector of the normalized effective capacities. We first have the following characterization.

Definition 1: The effective capacity region is described as

$$
\begin{aligned}
\mathcal{C}_{\mathrm{BC}}(\Theta)= & \bigcup_{\mathbf{R}}\{\mathrm{R}(\mathrm{R}\}) \geq \mathbf{0}: \\
& \left.\boldsymbol{C}_{j}\left(\theta_{j}\right) \leq-\frac{1}{\theta_{j} T B} \log _{e} \mathbb{E}_{\mathbf{z}}\left\{e^{-\theta_{j} T R_{j}}\right\}\right\}
\end{aligned}
$$

where $\mathbf{R}=\left\{R_{1}, R_{2}, \cdots, R_{M}\right\}$ represents the vector composed of the instantaneous transmission (or equivalently service) rates of $M$ users. Note that the union is over the distributions of the vector $\mathbf{R}$ such that the expected value $\mathbb{E}\{\mathbf{R}\}$ lies in the $\mathrm{BC}$ ergodic capacity region $\mathcal{R}_{\mathrm{BC}}$ given in (2) and power allocation policies satisfy the average power constraint (1).

The effective capacity region given in Definition 1 represents the set of all vectors of constant arrival rates $\mathrm{C}(\Theta)$ that can be supported in the fading broadcast channel in the presence QoS constraints specified by $\Theta=\left(\theta_{1}, \cdots, \theta_{M}\right)$. Since reliable communication is considered, arrival rates are supported by instantaneous service rates whose expected values are in the $\mathrm{BC}$ capacity region.

Using the convexity of the $\mathrm{BC}$ ergodic capacity region $\mathcal{R}_{\mathrm{BC}}$, we obtain the following preliminary result on the effective capacity region defined in (10).

Proposition 1: The effective capacity region $\mathcal{C}_{\mathrm{BC}}(\Theta)$ is convex.

Proof: Let the vectors $\mathrm{C}(\Theta)$ and $\mathrm{C}^{\prime}(\Theta)$ belong to $\mathcal{C}_{\mathrm{BC}}(\Theta)$. Then, there exist some rate vectors $\mathbf{R}$ and $\mathbf{R}^{\prime}$ for $C(\Theta)$ and $C^{\prime}(\Theta)$, respectively, such that $\mathbb{E}\{\mathbf{R}\}$ and $\mathbb{E}\left\{\mathbf{R}^{\prime}\right\}$ are in the $\mathrm{BC}$ ergodic capacity region. By a time sharing strategy, for any $\alpha \in(0,1)$, we know from the convexity of the BC ergodic capacity region that $\mathbb{E}\left\{\alpha \mathbf{R}+(1-\alpha) \mathbf{R}^{\prime}\right\} \in \mathcal{R}_{\mathrm{BC}}$. Now, we can write

$$
\begin{aligned}
& \alpha \mathrm{C}(\Theta)+(1-\alpha) \mathrm{C}^{\prime}(\Theta) \\
& \leq-\frac{1}{\Theta T B} \log _{e}\left(\mathbb{E}\left\{e^{-\Theta T \mathbf{R}}\right\}\right)^{\alpha}\left(\mathbb{E}\left\{e^{-\Theta T \mathbf{R}^{\prime}}\right\}\right)^{1-\alpha}
\end{aligned}
$$

$$
\begin{array}{rl}
=-\frac{1}{\Theta T B} \log _{e} & \left(\mathbb{E}\left\{\left(e^{-\Theta T \alpha \mathbf{R}}\right)^{\frac{1}{\alpha}}\right\}\right)^{\alpha} \\
& \times\left(\mathbb{E}\left\{\left(e^{-\Theta T(1-\alpha) \mathbf{R}^{\prime}}\right)^{\frac{1}{1-\alpha}}\right\}\right)^{1-\alpha} \\
\leq-\frac{1}{\Theta T B} \log _{e} & \mathbb{E}\left\{e^{-\Theta T\left(\alpha \mathbf{R}+(1-\alpha) \mathbf{R}^{\prime}\right)}\right\} .
\end{array}
$$

Above, in (11) through (13), all operations, including the logarithm and exponential functions and expectations, are component-wise operations. For instance, the expression in (11) denotes a vector whose components are $\left\{\frac{1}{\theta_{j} T B} \log _{e}\left(\mathbb{E}\left\{e^{-\theta_{j} T R_{j}}\right\}\right)^{\alpha}\left(\mathbb{E}\left\{e^{-\theta T R_{j}^{\prime}}\right\}\right)^{1-\alpha}\right\}_{j=1}^{M}$. Similarly, the inequalities in (11) and (13) are component-wise inequalities. The inequality in (11) follows from the definition in (10). (13) follows from Hölder's inequality and leads to the conclusion that $\alpha \mathrm{C}+(1-\alpha) \mathrm{C}^{\prime}$ still lies in the effective capacity region, proving the convexity result.

We are interested in the boundary of the region $\mathcal{C}_{\mathrm{BC}}(\Theta)$. Now that $\mathcal{C}_{\mathrm{BC}}(\Theta)$ is convex, we can characterize the boundary surface by considering the following optimization problem [1]:

$$
\max \lambda \cdot \mathrm{C}(\Theta) \text { subject to: } \mathrm{C}(\Theta) \in \mathcal{C}_{\mathrm{BC}}(\Theta) .
$$

for all priority vectors $\lambda=\left(\lambda_{1}, \cdots, \lambda_{M}\right)$ in $\mathfrak{R}_{+}^{M}$ with $\sum_{j=1}^{M} \lambda_{j}=1$.

1) Two-user Case: We know that the decoding order is given by the effective noise level. Suppose $\gamma=\frac{N_{2}}{N_{1}}$. Let $\mathcal{Z}=$ $\left\{\mathbf{z}: \gamma z_{1}>z_{2}\right\}$ be the region in which user 1 has less effective noise level and hence, it can first decode and eliminate the message intended for user 2 , and hence sees no interference. Then, $\mathcal{Z}^{c}=\left\{\mathbf{z}: \gamma z_{1}<z_{2}\right\}$ represents the region in which user 1 decodes its signal in the presence of interference from user 2's signal. In this scenario, we can write the maximum instantaneous rate for user 1 as

$$
R_{1}=\left\{\begin{array}{ll}
B \log _{2}\left(1+\mu_{1}(\mathbf{z}) z_{1}\right), & \mathbf{z} \in \mathcal{Z} \\
B \log _{2}\left(1+\frac{\mu_{1}(\mathbf{z}) z_{1}}{1+\gamma \mu_{2}(\mathbf{z}) z_{1}}\right), & \mathbf{z} \in \mathcal{Z}^{c}
\end{array},\right.
$$

and the instantaneous rate for user 2 as

$$
R_{2}=\left\{\begin{array}{ll}
B \log _{2}\left(1+\mu_{2}(\mathbf{z}) z_{2}\right), & \mathbf{z} \in \mathcal{Z}^{c} \\
B \log _{2}\left(1+\frac{\mu_{2}(\mathbf{z}) z_{2}}{1+\mu_{1}(\mathbf{z}) z_{2} / \gamma}\right) & \mathbf{z} \in \mathcal{Z}
\end{array} .\right.
$$

Now, using these instantaneous service rates $R_{1}$ and $R_{2}$ in the effective capacity expressions and recalling the average SNR constraint in (1), we can express the Lagrangian of the convex optimization problem in (14) as

$$
\begin{aligned}
\mathcal{J}=-\frac{\lambda_{1}}{\beta_{1} \log _{e} 2} & \log _{e}\left(\int_{\mathbf{z} \in \mathcal{Z}}\left(1+\mu_{1}(\mathbf{z}) z_{1}\right)^{-\beta_{1}} p_{\mathbf{z}}\left(z_{1}, z_{2}\right) d \mathbf{z}\right. \\
& \left.+\int_{\mathbf{z} \in \mathcal{Z}^{c}}\left(1+\frac{\mu_{1}(\mathbf{z}) z_{1}}{1+\gamma \mu_{2}(\mathbf{z}) z_{1}}\right)^{-\beta_{1}} p_{\mathbf{z}}\left(z_{1}, z_{2}\right) d \mathbf{z}\right) \\
-\frac{\lambda_{2}}{\beta_{2} \log _{e} 2} & \log _{e}\left(\int_{\mathbf{z} \in \mathcal{Z}^{c}}\left(1+\mu_{2}(\mathbf{z}) z_{2}\right)^{-\beta_{2}} p_{\mathbf{z}}\left(z_{1}, z_{2}\right) d \mathbf{z}\right. \\
& \left.+\int_{\mathbf{z} \in \mathcal{Z}}\left(1+\frac{\mu_{2}(\mathbf{z}) z_{2}}{1+\mu_{1}(\mathbf{z}) z_{2} / \gamma}\right)^{-\beta_{2}} p_{\mathbf{z}}\left(z_{1}, z_{2}\right) d \mathbf{z}\right) \\
& -\kappa\left(\mathbb{E}_{\mathbf{z}}\left\{\mu_{1}+\gamma \mu_{2}\right\}-\mathrm{SNR}\right)
\end{aligned}
$$


where $\beta_{j}=\frac{\theta_{j} T B}{\log _{e} 2}$ for $j=1,2, p_{\mathbf{z}}\left(z_{1}, z_{2}\right)$ is the joint distribution function of the fading states $\mathbf{z}=\left(z_{1}, z_{2}\right)$, and $\kappa \geq 0$ is the Lagrange multiplier. Next, we define $\left(\phi_{1}, \phi_{2}\right)$ as

$$
\begin{aligned}
\phi_{1}=\int_{\mathbf{z} \in \mathcal{Z}}(1 & \left.+\mu_{1}(\mathbf{z}) z_{1}\right)^{-\beta_{1}} p_{\mathbf{z}}\left(z_{1}, z_{2}\right) d \mathbf{z} \\
& +\int_{\mathbf{z} \in \mathcal{Z}^{c}}\left(1+\frac{\mu_{1}(\mathbf{z}) z_{1}}{1+\gamma \mu_{2}(\mathbf{z}) z_{1}}\right)^{-\beta_{1}} p_{\mathbf{z}}\left(z_{1}, z_{2}\right) d \mathbf{z}, \\
\phi_{2}=\int_{\mathbf{z} \in \mathcal{Z}^{c}}(1 & \left.+\mu_{2}(\mathbf{z}) z_{2}\right)^{-\beta_{2}} p_{\mathbf{z}}\left(z_{1}, z_{2}\right) d \mathbf{z} \\
& +\int_{\mathbf{z} \in \mathcal{Z}}\left(1+\frac{\mu_{2}(\mathbf{z}) z_{2}}{1+\mu_{1}(\mathbf{z}) z_{2} / \gamma}\right)^{-\beta_{2}} p_{\mathbf{z}}\left(z_{1}, z_{2}\right) d \mathbf{z} .
\end{aligned}
$$

Below, we derive the optimality conditions (that the optimal power control policies should satisfy) by differentiating the Lagrangian with respect to $\mu_{1}, \mu_{2}$ in regions $\mathcal{Z}^{c}$ and $\mathcal{Z}$, and making the derivatives equal to zero:

1) $\begin{aligned} & \frac{\lambda_{1}}{\phi_{1} \log _{e} 2}\left(1+\mu_{1} z_{1}\right)^{-\beta_{1}-1} z_{1}-\frac{\lambda_{2}}{\phi_{2} \log _{e} 2} \\ & \times\left(1+\frac{\mu_{2} z_{2}}{1+\mu_{1} z_{2} / \gamma}\right)^{-\beta_{2}-1} \frac{\mu_{2} z_{2}^{2} / \gamma}{\left(1+\mu_{1} z_{2} / \gamma\right)^{2}}-\kappa=0 \quad \forall \mathbf{z} \in \mathcal{Z}\end{aligned}$

2) $\frac{\lambda_{2}}{\phi_{2} \log _{e} 2}\left(1+\frac{\mu_{2} z_{2}}{1+\mu_{1} z_{2} / \gamma}\right)^{-\beta_{2}-1} \frac{z_{2}}{1+\mu_{1} z_{2} / \gamma}-\gamma \kappa=0$

3) $\frac{\lambda_{1}}{\phi_{1} \log _{e} 2}\left(1+\frac{\mu_{1} z_{1}}{1+\gamma \mu_{2} z_{1}}\right)^{-\beta_{1}-1} \frac{z_{1}}{1+\gamma \mu_{2} z_{1}}-\kappa=0 \quad \forall \mathbf{z} \in \mathcal{Z}^{c}$

$$
\begin{aligned}
4)- & \frac{\lambda_{1}}{\phi_{1} \log _{e} 2}\left(1+\frac{\mu_{1} z_{1}}{1+\gamma \mu_{2} z_{1}}\right)^{-\beta_{1}-1} \frac{\mu_{1} \gamma z_{1}^{2}}{\left(1+\gamma \mu_{2} z_{1}\right)^{2}} \\
& +\frac{\lambda_{2}}{\phi_{2} \log _{e} 2}\left(1+\mu_{2} z_{2}\right)^{-\beta_{2}-1} z_{2}-\gamma \kappa=0 \quad \forall \mathbf{z} \in \mathcal{Z}^{c} .
\end{aligned}
$$

Above, (17) through (20) are obtained by evaluating the derivative of $\mathcal{J}$ with respect to $\mu_{1}$ when $\mathbf{z} \in \mathcal{Z}, \mu_{2}$ when $\mathbf{z} \in \mathcal{Z}, \mu_{1}$ when $\mathbf{z} \in \mathcal{Z}^{c}$, and $\mu_{2}$ when $\mathbf{z} \in \mathcal{Z}^{c}$, respectively. Whenever $\mu_{1}$ or $\mu_{2}$ turns out to have negative values through these equations, they are set to 0 due to the convexity of the optimization problem [10].

We immediately note from (18) that when $\mathbf{z} \in \mathcal{Z}$, the optimal power control policy for receiver 2 can be expressed as

$$
\mu_{2}=\left[\frac{1}{\alpha_{2}^{\frac{1}{\beta_{2}+1}}\left(\frac{z_{2}}{1+\mu_{1} z_{2} / \gamma}\right)^{\frac{\beta_{2}}{\beta_{2}+1}}}-\frac{1+\mu_{1} z_{2} / \gamma}{z_{2}}\right]^{+} \quad \forall \mathbf{z} \in \mathcal{Z}
$$

where $\alpha_{2}=\frac{\gamma \kappa \phi_{2} \log _{e} 2}{\lambda_{2}}$. We can see from (21) that when $\mu_{1}>$ $\gamma\left(\frac{1}{\alpha_{2}}-\frac{1}{z_{2}}\right)$, we have $\mu_{2}=0$. Also, when $\mathbf{z} \in \mathcal{Z}$, if no power is allocated for user 2 and hence $\mu_{2}=0$, then we see from (17) that we can write the optimal $\mu_{1}$ as

$$
\mu_{1}=\left[\frac{1}{\alpha_{1}^{\frac{1}{\beta_{1}+1}} z_{1}^{\frac{\beta_{1}}{\beta_{1}+1}}}-\frac{1}{z_{1}}\right]^{+} \quad \forall \mathbf{z} \in \mathcal{Z}
$$

where $\alpha_{1}=\frac{\kappa \phi_{1} \log _{e} 2}{\lambda_{1}}$. Note also that when messages for both receivers are being transmitted simultaneously, $\mu_{1}$ and $\mu_{2}$ are the positive solutions to (17) and (18). This, after straightforward derivations, implies that the equation

$$
\frac{z_{1}}{\alpha_{1}}\left(1+\mu_{1} z_{1}\right)^{-\beta_{1}-1}-\left(\frac{z_{2}}{\alpha_{2}\left(1+\mu_{1} z_{2} / \gamma\right)}\right)^{\frac{1}{\beta_{2}+1}}=0
$$

has a positive solution that is less than $\gamma\left(\frac{1}{\alpha_{2}}-\frac{1}{z_{2}}\right)$.

Following similar steps, we can characterize the power control policies for the case in which $\mathbf{z} \in \mathcal{Z}^{c}$. In this case, it is worth noting that the condition for having $\mu_{1}>0$ and $\mu_{2}>0$ is now that the equation

$$
\frac{z_{2}}{\alpha_{2}}\left(1+\mu_{2} z_{2}\right)^{-\beta_{2}-1}-\left(\frac{z_{1}}{\alpha_{1}\left(1+\gamma \mu_{2} z_{1}\right)}\right)^{\frac{1}{\beta_{1}+1}}=0
$$

has a positive solution that is less than $\frac{1}{\gamma}\left(\frac{1}{\alpha_{1}}-\frac{1}{z_{1}}\right)$. This can be regarded as the symmetric case of (23).

As seen in the above discussion, we have no closed-form expressions for the optimal power control policies which are in general interdependent on each other. The optimal power control policies can be determined through numerical computations. Thus, we propose the algorithm below to obtain the optimal power adaptation policies. This algorithm is used in the numerical results presented in Section IV-C.

\section{POWER CONTROL Algorithm}

Given $\lambda_{0}, \lambda_{1}$, initialize $\phi_{1}, \phi_{2}$

2 Initialize $\kappa$;

Determine $\alpha_{1}=\frac{\kappa \phi_{1} \log _{e} 2}{\lambda_{1}}, \alpha_{2}=\frac{\gamma \kappa \phi_{2} \log _{e} 2}{\lambda_{2}}$;

if $\gamma z_{1}>z_{2}$

then if $z_{1}>\alpha_{1}$

then Compute $\mu_{1}$ from (22);

if $\mu_{1}>\gamma\left(\frac{1}{\alpha_{2}}-\frac{1}{z_{2}}\right)$ or $z_{2}<\alpha_{2}$

then $\mu_{2}=0$;

else if (23) returns positive solution

then Compute $\mu_{1}$ and $\mu_{2}$ from (17) and (18);

else $\mu_{1}=0, \mu_{2}=\left[\frac{1}{\alpha_{2}^{\frac{1}{\beta_{2}+1}} z_{2}^{\frac{\beta_{2}}{\beta_{2}+1}}}-\frac{1}{z_{2}}\right]^{+}$;

else

if $z_{2}>\alpha_{2}$

$$
\begin{aligned}
& \text { then } \mu_{2}=\frac{1}{\frac{1}{\alpha_{2}^{\beta_{2}+1}} \frac{\beta_{2}}{\beta_{2}+1}}-\frac{1}{z_{2}} ; \\
& \text { if } \mu_{2}>\frac{1}{\gamma}\left(\frac{1}{\alpha_{1}}-\frac{1}{z_{1}}\right) \text { or } z_{1}<\alpha_{1} \\
& \text { then } \mu_{1}=0 ; \\
& \text { else if (24)returns positive solution }
\end{aligned}
$$

then Compute $\mu_{1}$ and $\mu_{2}$ from (20) and (19);

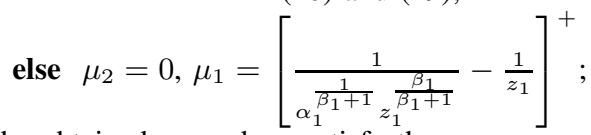

Check if the obtained $\mu_{1}$ and $\mu_{2}$ satisfy the average power constraint with equality;

if not satisfied with equality

then update the value of $\kappa$ and return to Step 3; else move to Step 27;

Evaluate $\phi_{1}$ and $\phi_{2}$ with the obtained power control policies;

Check if the new values of $\phi_{1}$ and $\phi_{2}$ agree (up to a certain margin) with those used in Step 3;

if do not agree

then update the values of $\phi_{1}$ and $\phi_{2}$ and return to Step 2; else declare the obtained power allocation policies $\mu_{1}$ and $\mu_{2}$ as the optimal ones. 


\section{B. Time-Division Multiplexing}

In this section, we discuss the effective throughtput region achieved with the TDM strategy described in Section II-B. Now, substituting (4) into (8) and normalizing the effective rate with the bandwidth $B$ give us the effective capacity for each receiver as

$$
\mathrm{C}_{j}=-\frac{1}{\beta_{j} \log _{e} 2} \log _{e}\left(\int_{\mathbf{z}}\left(1+\mu_{j}(\mathbf{z}) z_{j}\right)^{-\delta_{j} \beta_{j}} p_{\mathbf{z}}(\mathbf{z}) d \mathbf{z}\right)
$$

where we again have $\beta_{j}=\frac{\theta_{j} T B}{\log _{e} 2}$ and $j=1,2, \ldots, M$. Above, we see that through the use of this TDM scheme, we have $C_{j}$ depend only on $\mu_{j}$. At the same time, note that we still have the constraint (1).

In order to identify the effective throughput region achieved with TDM, we employ the following two steps. We initially decouple the problem of finding the optimal power control policies $\mu_{j}$ by fixing the average power and time allocated to each receiver. As can be seen, the optimal power control policy is given by [5]

$$
\mu_{j}^{*}(\mathbf{z})=\left[\frac{1}{\alpha_{j}^{\frac{1}{\rho_{j} \beta_{j}+1}} z_{j}^{\frac{\rho_{j} \beta_{j}}{\rho^{\beta_{j}+1}}}}-\frac{1}{z_{j}}\right]^{+}
$$

where $\alpha_{j}$ is chosen to satisfy the average power constraint (5). Subsequently, we identify the effective capacities achieved with different average power $\rho=\left(\rho_{1}, \ldots, \rho_{M}\right)$ and time allocations $\delta=\left(\delta_{1}, \ldots, \delta_{M}\right)$. The union of the effective capacity $M$-tuples over all possible time and power allocations provide the throughput region.

\section{Numerical Results}

In Fig. 2, we provide the effective capacity region achieved with superposition coding and the effective throughput region attained with TDM in a Rayleigh fading environment in which $z_{1}$ and $z_{2}$ are independent exponential random variables with $\mathbb{E}\left\{z_{1}\right\}=\mathbb{E}\left\{z_{2}\right\}=1$. We assume $\gamma=1$, SNR $=-10 \mathrm{~dB}$, and $\theta_{1}=\theta_{2}=0.01$. $T=2 \mathrm{~ms}, B=10^{5} \mathrm{~Hz}$. The solid line provides the boundary region of the superposition scheme with optimal power control policies. The inner region represents the maximum effective capacity pairs for different values of $\delta$ and $\rho$ in the TDM scheme. Union of all the points correspond to the effective throughput region of TDM. In the figure, we observe that TDM is always inside the optimal effective capacity region, and hence is suboptimal.

\section{CONCLUSION}

In this paper, we have investigated the performance of transmission over fading broadcast channels in the presence of statistical QoS constraints. We have first defined the effective capacity region when superposition coding with successive interference cancellation is used and proved that this region is convex. Then, we have obtained the optimal power control policies that achieve the points on the boundary region for the two-user case. In particular, we have identified an algorithm for computing the optimal power allocated to each fading state

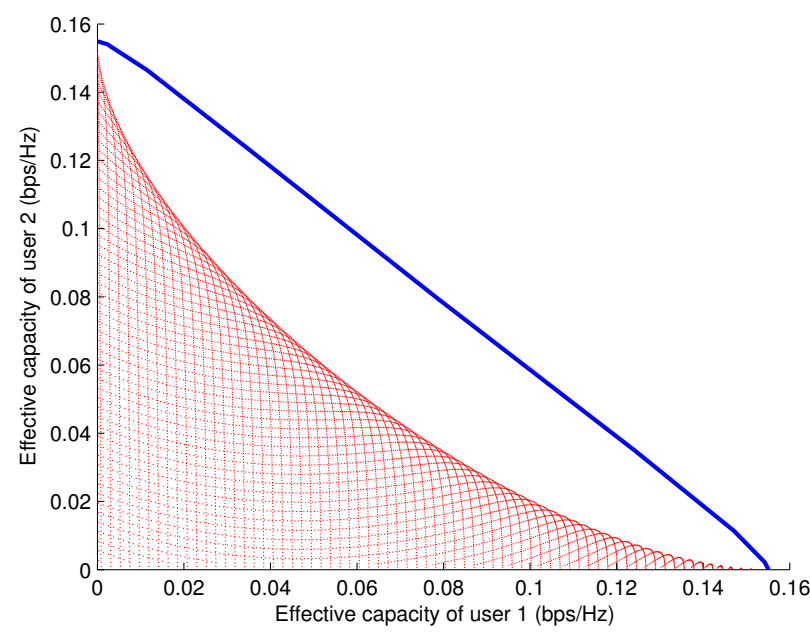

Fig. 2. The effective capacity region of the two-user fading broadcast channel (boundary given by the solid blue line), and the effective throughput region of the TDM scheme (inner region described by the union of the red lines).

using the optimality conditions. Also, we have characterized the effective throughput region achieved by a simple timedivision multiplexing scheme. Through numerical results, we have noted that superposition coding with successive decoding can lead to improvements in the region when compared to that attained by orthogonal transmission schemes such as TDM.

\section{REFERENCES}

[1] L. Li and A. Goldsmith, "Capacity and optimal resource allocation for fading broadcast channels-Part I: Ergodic capacity," IEEE Trans. Inform. Theory, vol. 47, no. 3, pp. 1083-1102, March 2001.

[2] L. Li and A. Goldsmith, "Capacity and optimal resource allocation for fading broadcast channels-Part II: Outage capacity," IEEE Trans. Inform. Theory, vol. 47, no. 3, pp. 1103-1127, March 2001.

[3] K. Seong, R. Narasimhan, and J. M. Cioffi, "Queue proportional scheduling via Geometric programming in fading broadcast channels," IEEE J. Select. Areas Commun., vol. 24, no. 8, pp. 1593-1602, Aug. 2006.

[4] D. Wu and R. Negi "Effective capacity: a wireless link model for support of quality of service," IEEE Trans. Wireless Commun., vol.2,no. 4, pp.630-643. July 2003.

[5] J. Tang and X. Zhang, "Quality-of-service driven power and rate adaptation over wireless links," IEEE Trans. Wireless Commun., vol. 6, no. 8, pp. 3058-3068, Aug. 2007.

[6] J. Tang and X. Zhang, "Cross-layer-model based adaptive resource allocation for statistical QoS guarantees in mobile wireless networks,' IEEE Trans. Wireless Commun., vol. 7, no. 6, pp.2318-2328, June 2008.

[7] L. Liu, P. Parag, and J.-F. Chamberland, "Quality of service analysis for wireless user-cooperation networks," IEEE Trans. Inform. Theory, vol. 53, no. 10 , pp. 3833-3842, Oct. 2007

[8] Q. Du and X. Zhang, "Resource allocation for downlink statistical multiuser QoS provisionings in cellular wireless networks," Proc. of IEEE INFOCOM 2008, April 2008.

[9] A. Balasubramanian and S.L. Miller, "Effective capacity of a time division downlink scheduling system,"IEEE Trans. Commun., vol. 58, no. 1, pp. 73-78, Jan. 2010.

[10] S. Boyd and L. Vandenberghe, Convex Optimization, Cambridge University Press, 2004. 